\title{
Tyrosine and aurora kinase inhibitors diminish transport function of multidrug resistance-associated protein (MRP) 4 and breast cancer resistance protein (BCRP)
}

\author{
Rhiannon N. Hardwick ${ }^{1}$, Marina Snellings ${ }^{2}$, Brian C. Ferslew ${ }^{2}$, Yang Lu ${ }^{2}$, Kim L.R. \\ Brouwer $^{1,2^{*}}$ \\ ${ }^{1}$ Curriculum in Toxicology, UNC School of Medicine, North Carolina, US \\ ${ }^{2}$ Division of Pharmacotherapy and Experimental Therapeutics, UNC Eshelman School of Pharmacy, The University of \\ North Carolina at Chapel Hill, US
}

*Corresponding Author: E-mail: kbrouwer@unc.edu ; Tel.: +1-919-962-7030; Fax: +1-919-962-0644

Received: June 27, 2016; Revised: December 07, 2016; Published: December 26, 2016

\begin{abstract}
Tyrosine and aurora kinases are important effectors in signal transduction pathways that are often involved in aberrant cancer cell growth. Tyrosine (TKI) and aurora (AKI) kinase inhibitors are anti-cancer agents specifically designed to target such signaling pathways through TKI/AKI binding to the ATP-binding pocket of kinases thereby leading to diminished kinase activity. Some TKIs have been identified as inhibitors of ATP-binding cassette $(A B C)$ transporters such as $P$-glycoprotein and breast cancer resistance protein $(B C R P)$, which are commonly upregulated in malignant cells. TKI/AKIs have been investigated as $A B C$ transporter inhibitors in order to facilitate the accumulation of concomitantly administered chemotherapeutics within cancer cells. However, $A B C$ transporters are prominently expressed in the liver and other eliminating organs, and their inhibition has been linked to intracellular accumulation of drugs, altered disposition, and toxicity. The potential for TKIs/AKIs to inhibit other important hepatic efflux transporters, particularly multidrug resistance-associated proteins (MRPs), remains unknown. The aim of the current study was to compare the inhibitory potency of 20 selected TKI/AKIs against MRP4 and BCRP through the use of inverted membrane vesicle assays. Relative $I C_{50}$ values were estimated by determining TKI/AKI inhibition of MRP4-mediated [ ${ }^{3} \mathrm{H}$ ]-dehydroepiandrosterone sulfate uptake and BCRP-mediated [ ${ }^{3} \mathrm{H}$ ]estrone sulfate uptake. To provide insight to the clinical relevance of TKI/AKI inhibition of $A B C$ efflux transporters, the ratio of the steady-state maximum total plasma concentration $\left(C_{s s}\right)$ to the $I C_{50}$ for each compound was calculated with $C_{s s} / I C_{50}$ ratio $>0.1$ deemed potentially clinically relevant. Such analysis identified several potentially clinically relevant inhibitors of MRP4: alisertib, danusertib, erlotinib, lapatinib, neratinib, nilotinib, pazopanib, sorafenib, and tozasertib. The potentially clinically relevant inhibition of $B C R P$ was much more extensive and included alisertib, barasertib, danusertib, enzastaurin, erlotinib, gefitinib, imatinib, neratinib, nilotinib, pazopanib, selumetinib, sorafenib, sunitinib, tozasertib, and vandetanib. These findings indicate the significant potential for TKI/AKIs to inhibit multiple ABC efflux transporters. The resulting inhibition data could provide insight regarding the clinical interpretation of pharmacokinetic/pharmacodynamic outcomes when TKI/AKIs are administered concomitantly with additional chemotherapeutic agents.
\end{abstract}

\section{Keywords}

$A B C$ transporters; transport inhibition 


\section{Introduction}

Small molecule kinase inhibitors have revolutionized the field of oncology by facilitating the targeting of specific kinases, which have been found to be over-expressed and/or mutated in specific tumor types. Such targeting has enabled a more directed, individualistic treatment regimen for many patients [1-4]. These small molecule kinase inhibitors have been designed primarily to target specific receptor tyrosine kinases, namely the epidermal growth factor receptor (EGFR and HER2), vascular endothelial growth factor receptor (VEGFR), platelet-derived growth factor receptor (PDGFR), and the BCR/ABL (breakpoint cluster region/Abelson) fusion protein; however, some off-target inhibition of intracellular kinases can occur. Receptor tyrosine kinases are important mediators of intracellular signaling cascades [4-6]. Specifically, upon binding of ligand to the receptor tyrosine kinase, autocatalytic phosphorylation events spark the propagation of one or more signaling cascades ultimately leading to transcription of genes. Aberrant regulation of receptor tyrosine kinases can result in amplification of genes related to proliferation, apoptosis, and differentiation, and thus enable cancer cell growth and metastasis [3,6]. Aurora kinases are different from receptor tyrosine kinases in that they are primarily active during mitosis, but also have been found to be upregulated in many tumor types $[7,8]$. As such, several small molecule inhibitors have been designed in an effort to mitigate the activity of aurora kinases during cancer cell growth. Though kinase inhibitors are designed with a particular target in mind, cross-reactivity with off-target kinases can occur in nonmalignant cells, which may increase the potential for off-target, potentially toxic effects $[4,9]$.

TKIs and AKIs exert their effects by acting as ATP-mimics and binding to the ATP-binding pocket of kinases to decrease the kinase catalytic activity [2]. In recent years, TKIs have been identified as inhibitors of several ATP-binding cassette $(A B C)$ efflux transporters that are commonly upregulated in cancer cells including P-glycoprotein (multidrug resistance protein 1), breast cancer resistance protein (BCRP), and multidrug resistance-associated protein (MRP) 1 [10-18]. ABC efflux transporters consist of a large family of membrane-spanning proteins involved in the active extrusion of substrates such as endogenous molecules, drugs, and drug metabolites from the cell through hydrolysis of ATP [19-21]. ABC efflux transporters are expressed prominently in eliminating organs such as the liver and kidney, and thus can play a significant role in the overall disposition of a drug within the body [19]. Upregulation of $A B C$ efflux transporters in cancer cells confers chemoresistance, and imparts a greater ability of the cell to limit exposure to, and diminish the effects of, chemotherapeutic agents $[12,22,23]$. The upregulation of $A B C$ transporters during chemoresistance has made them an attractive target in overcoming the clinical difficulty of maintaining intracellular exposure to chemotherapeutics. Due to their ability to inhibit $A B C$ transporters commonly upregulated in cancer cells, kinase inhibitors recently have been investigated for their capability to reverse chemoresistance. In particular, BCRP can confer resistance to gefitinib, but in contrast also is inhibited by gefitinib, vandetanib, and neratinib [18]. Similarly, it has been shown that sunitinib is an inhibitor of BCRP and can reverse chemoresistance to co-administered chemotherapeutics in BCRP over-expressing cells [16]. Others have shown that dasatinib, nilotinib, and imatinib are competitive inhibitors of P-glycoprotein and $B C R P$, meaning that they are substrates at low concentrations, while they inhibit transport function at high concentrations [24]. Although these data indicate the potential for kinase inhibitors to be used as tools to overcome chemoresistance through $A B C$ efflux transporter inhibition in vitro, several clinical trials employing kinase inhibitors for mitigation of chemoresistance have been unable to definitively demonstrate improvements in progression free survival and/or overall survival of patients [25-28].

Studies seeking to utilize kinase inhibitors for mitigation of chemoresistance though inhibition of specific $A B C$ efflux transporters that are upregulated in cancer cells often fail to address a key 
complication: off-target inhibition of $A B C$ efflux transporters in eliminating organs such as liver, kidney, and intestine. Several MRPs including MRP2, MRP3, and MRP4 are differentially localized to the apical and basolateral membranes of hepatocytes, enterocytes, and proximal tubule cells, which enables them to direct the disposition of some chemotherapeutics $[19,20]$. For example, MRP2 is localized to the apical membrane of hepatocytes while MRP3 is situated at the basolateral membrane. MRP2 and MRP3 share substrate specificity yet extrude drugs and drug metabolites into the bile and systemic circulation, respectively [19]. Inhibition of MRP2 can shift the hepatobiliary disposition of a drug from bile, and subsequent elimination in feces, to the sinusoidal blood resulting in greater systemic exposure. Similar to MRP3, MRP4 resides on the basolateral membrane of hepatocytes, while BCRP, like MRP2, is localized to the apical membrane with each differentially extruding drugs and metabolites into the systemic circulation and bile, respectively. MRP4 transports nucleosides, Phase II conjugation products, and conjugated bile acids $[20,29]$. Inhibition of MRP4-mediated transport has been associated with an increased risk of cholestatic potential, particularly among compounds that do not inhibit the bile salt export pump (BSEP) [29]. Similarities in the chemical properties of MRP4 inhibitors and BSEP inhibitors have been identified [30]. Collectively, data suggest that MRP4 inhibition may serve as a confounding factor in BSEP-mediated drug-induced liver injury or, in some cases, lead to drug-induced liver injury in the absence of BSEP inhibition. Thus, inhibition of one or more $A B C$ efflux transporters can significantly affect the disposition of a co-administered drug, and in some cases, may impact the risk of hepatotoxicity. Knowledge of the inhibitory potency of TKIs and AKIs for multiple ABC efflux transporters, not limited to those upregulated in cancer cells, is critically important to predict the pharmacokinetic and pharmacodynamic outcomes and potential for hepatotoxicity of TKI/AKIs and co-administered chemotherapeutics. The purpose of the current study was to compare the inhibitory potency of 20 TKIs and AKIs (see Table 1 for selected compounds) against the $A B C$ efflux transporters MRP4 and BCRP using inverted membrane vesicle assays. Additionally, half maximal inhibitory concentration $\left({ } C_{50}\right)$ data were leveraged against reported steady state concentration $\left(C_{S S}\right)$ values to provide insight to the clinical relevance of TKI/AKI inhibition of MRP4- and BCRP-mediated transport function.

Table 1. Selected Tyrosine (TKI) and Aurora (AKI) Kinase Inhibitors. Twenty TKI/AKIs were selected for analysis of their potential to inhibit MRP4- and BCRP-mediated transport. TKI/AKIs are categorized by primary kinase target. EGFR, epidermal growth factor receptor; VEGFR, vascular endothelial growth factor receptor; PDGFR, platelet-derived growth factor receptor; BCR/ABL, Breakpoint cluster region/Abelson fusion protein.

\begin{tabular}{|ll|}
\hline Primary Target & Inhibitors \\
EGFR & erlotinib, gefitinib, lapatinib, neratinib, vandetanib \\
VEGFR \& PDGFR & axitinib, pazopanib, sorafenib, sunitinib \\
BCR/ABL & bosutinib, dasatinib, imatinib, nilotinib \\
Miscellaneous & crizotinib (anaplastic lymphoma kinase), enzastaurin (protein kinase C isoforms), selumetinib \\
& (mitogen-activated protein kinase 1) \\
Aurora & alisertib (Aurora A), barasertib (Aurora B), danusertib (Aurora A/B/C), tozasertib (Aurora \\
& A/B/C) \\
\hline
\end{tabular}

\section{Experimental}

\section{Materials}

Cell culture supplies were obtained from Gibco (Life Technologies, Grand Island, NY), and hygromycin B from Roche Life Sciences (Indianapolis, IN). Microscint-20, [ $\left.{ }^{3} \mathrm{H}\right]$-dehydroepiandrosterone-sulfate (DHEAS; $70.5 \mathrm{Ci} / \mathrm{mmol}$ ) and $\left[{ }^{3} \mathrm{H}\right]$-estrone sulfate $(\mathrm{E} 1 \mathrm{~S} ; 45 \mathrm{Ci} / \mathrm{mmol})$ were purchased from Perkin Elmer Life and Analytical Sciences (Waltham, MA). DMSO (dimethylsulfoxide) was obtained from Fisher Scientific (Fairlawn, NH). Compounds were purchased from Selleckchem (Houston, TX) or Cayman Chemical (Ann Arbor, MI). 


\section{Cell Culture and Membrane Vesicle Preparation}

HEK293T cells stably overexpressing MRP4 (HEK-MRP4), HEK293T cells transfected with empty vector (HEK-MOCK), MDCKII cells stably overexpressing BCRP (MDCKII-BCRP), and untransfected MDCKII cells were cultured in DMEM supplemented with $10 \% \mathrm{FBS}$ as previously described [29, 31]. MRP4 or BCRP overexpression was maintained by antibiotic selection with hygromycin B [29]. Cells were grown to 85-95\% confluence before membrane vesicle preparation. Membrane vesicles were prepared as previously described [31-34]. Vesicles were validated for maximal activity and used in transport studies within 1-2 weeks of preparation.

\section{Western Blot Analysis of Transporter Expression}

Transporter over-expression was verified in control and transporter over-expressing membrane vesicles via western blot analysis. Lysates of membrane vesicles $(15 \mu \mathrm{g} /$ well) were prepared in tris-glycine SDS sample buffer (Life Technologies) and separated by SDS-polyacrylamide gel electrophoresis (SDS-PAGE) using 4-12 \% tris-glycine gels (Life Technologies). Proteins separated by SDS-PAGE were transferred to polyvinylidene difluoride (PVDF) membranes overnight. Protein levels on membranes were determined by incubation with antibodies directed against MRP4 $\left(\mathrm{M}_{4} \mathrm{l}-10\right.$, generously provided by George L. Scheffer, Amsterdam, The Netherlands) or BCRP (BXP-21, Kamiya Biomedical Company).

\section{Membrane Vesicle Transport Assay}

TKI- and AKI-mediated inhibition of transport function was investigated in membrane vesicles so as to study the function of a single transporter in isolation without confounding variables such as cellular uptake or protein binding. To confirm transport based on previously developed assays, transport of [ $\left.{ }^{3} \mathrm{H}\right]-$ DHEAS (2 $\mu \mathrm{M}, 0.7 \mu \mathrm{Ci} / \mathrm{mL})$ by MRP4 or $\left.{ }^{3} \mathrm{H}\right]-\mathrm{E} 1 \mathrm{~S}(1 \mu \mathrm{M} ; 0.4 \mu \mathrm{Ci} / \mathrm{mL})$ by BCRP was measured over time via incubation of control (HEK-MOCK or MDCKII) and transporter over-expressing (HEK-MRP4 or MDCKII-BCRP) membrane vesicles in tris-sucrose buffer $(50 \mathrm{mM}$ tris/ $\mathrm{HCl}$ and $250 \mathrm{mM}$ sucrose, $\mathrm{pH}$ 7.4) in the presence or absence of an ATP-regenerating system ( $4 \mathrm{mM}$ ATP, $10 \mathrm{mM} \mathrm{MgCl}_{2}, 10 \mathrm{mM}$ creatine phosphate, and 0.1 $\mathrm{mg} / \mathrm{mL}$ creatine kinase). Transporter substrate concentrations were selected based on $\mathrm{Km}$ values [29, 34]. AMP was replaced with ATP for incubations conducted in the absence of an ATP-regenerating system. The difference between uptake with ATP versus AMP was used to determine the ATP-dependent uptake of $\left[{ }^{3} \mathrm{H}\right]-$ DHEAS and $\left[{ }^{3} \mathrm{H}\right]-\mathrm{E} 1 \mathrm{~S}$ in MRP4 and BCRP membrane vesicles, respectively. For transport inhibition assays, 20 different TKI- or AKI-test compounds (alisertib, axitinib, barasertib, bosutinib, crizotinib, danusertib, dasatinib, enzastaurin, erlotinib, gefitinib, imatinib, lapatinib, neratinib, nilotinib, pazopanib, selumetinib, sorafenib, sunitinib, tozasertib, or vandetanib) solubilized in DMSO (1 \% DMSO final reaction concentration) were added in separate experiments to the reaction mixture containing control or transporter over-expressing membrane vesicles in tris-sucrose buffer with the ATP-regenerating system. Test compounds were examined at a concentration range of 0.316-100 $\mu \mathrm{M}$. For potent BCRP inhibitors, the concentration range was reduced to $0.00316 \mu \mathrm{M}$. For control samples, identical concentrations of the solvent ( $1 \% \mathrm{DMSO}$ ) were used. All uptake studies were performed at $37{ }^{\circ} \mathrm{C}$. Uptake was stopped by addition of ice cold tris-sucrose buffer followed by rapid vacuum filtration through glass fiber 96 -well plates pre-soaked in tris-sucrose buffer (BCRP reactions) or tris-sucrose buffer with $3 \mathrm{mM}$ glutathione and $10 \mathrm{mM}$ dithiothreitol (MRP4). Microscint-20 (75 $\mu \mathrm{L}$ ) was added to each well and the plate was allowed to rotate on an orbital shaker for 15 minutes. Radioactivity was quantified by liquid scintillation counting in a TopCount NXT instrument (Perkin Elmer). Incubations with test compounds were completed in at least three independent experiments with no replicates in individual experiments. Positive control inhibitors (MK571 
for MRP4; sulfasalazine and promethazine for BCRP) were included in each individual experiment for quality control.

\section{Data Analysis and Statistics}

Substrate uptake into vesicles was calculated as pmol/mg protein/min before conversion to percent of control inhibition. Inhibition by TKI/AKIs as a percentage of control was calculated as the difference between substrate uptake in control and transporter-overexpressing membrane vesicles compared to vehicle-treated membrane vesicles (mean \pm standard deviation, SD). Dose-response IC $_{50}$ curves were fit via nonlinear regression analysis according to the four-parameter logistic model (4PL) with the bottom constraint set to $>0$ using GraphPad Prism 5. For analysis of potential clinical relevance of transporter inhibition, steady-state total plasma $C_{s s}$ values for each TKI/AKI were extracted from Micromedex and manufacturer package inserts.

\section{Results and Discussion}

MRP4 over-expression was confirmed in HEK-MRP4 membrane vesicles, and was significantly higher than HEK-MOCK membrane vesicles (Figure 1A). The time- and ATP-dependent uptake of [ $\left.{ }^{3} \mathrm{H}\right]-\mathrm{DHEAS}$ in HEK-MRP4 vesicles was linear up to 10 minutes (Figure $1 A$ ), similar to previous studies [29]. [ ${ }^{3} \mathrm{H}$ ]-DHEAS uptake in HEK-MRP4 vesicles was at least 75-fold greater than in HEK-MOCK vesicles. An incubation time of 3.5 minutes was chosen for experiments with HEK-MRP4 vesicles to evaluate the inhibition potential of test compounds, similar to previous studies [29]. The pan-MRP inhibitor MK571 was assessed across a concentration range of 0.316-100 $\mu \mathrm{M}$. MK571 produced a prototypical dose-response curve ranging from nearly full activity to complete inhibition (Figure 1B) with an $\mathrm{IC}_{50}$ value of $2.04 \mu \mathrm{M}$ (95\% confidence interval: 1.31-3.19 $\mu \mathrm{M})$. Morgan, et al. previously reported an $\mathrm{IC}_{50}$ value of $6 \mu \mathrm{M}$ for MK571-mediated inhibition of ${ }^{3} \mathrm{H}$-estradiol-17 $\beta$-D-glucuronide uptake in MRP4 membrane vesicles derived from Sf9 cells [35]. MK571 was included as a positive control with all subsequent test compound experiments for quality assurance of the MRP4 vesicle assay.

(A)

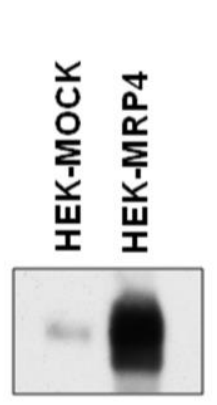

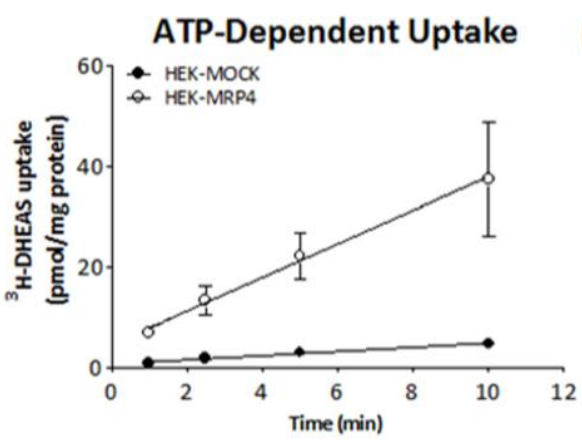

(B)

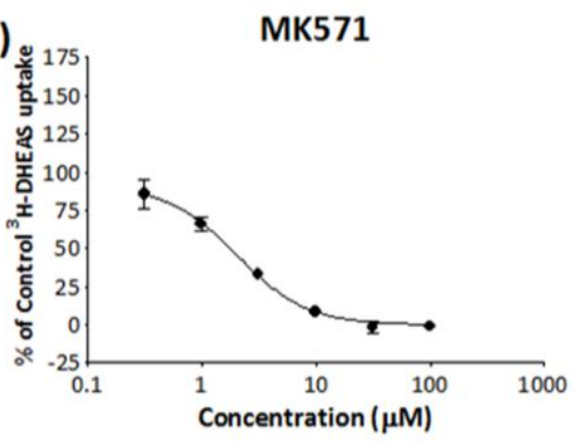

Figure 1. Characterization of the MRP4 Membrane Vesicle Assay. (A) MRP4 expression in HEK-MOCK and HEK-MRP4 cells. Also shown is ATP-dependent, time-dependent uptake of $2 \mu \mathrm{M}\left[{ }^{3} \mathrm{H}\right]$-dehydroepiandrosterone

(DHEAS) in membrane vesicles. Data are presented as mean $\pm S D, n=3$ independent experiments. (B) Doseresponse $\mathrm{IC}_{50}$ curve of the MRP4 positive control inhibitor MK571 (3.5 min incubation). Data are presented as mean $\%$ of control uptake $\pm S D, n=7$ independent experiments.

The inhibition potential of $20 \mathrm{TKI} / \mathrm{AKIs}$ was assessed in control and MRP4 over-expressing membrane vesicles. Dose-response curves of TKIs are shown in Figure 2. Of the 16 TKIs examined, 6 exhibited IC $\mathrm{C}_{50}$ values $<10 \mu \mathrm{M}$ (Table 2), namely, the EGFR inhibitors erlotinib, gefitinib, lapatinib and neratinib; the VEGFR \& PDGFR inhibitor sorafenib; and the BCR/ABL inhibitor nilotinib. Others have reported the inhibitory 
potency of some TKIs against MRP4-mediated transport, particularly dasatinib, gefitinib, imatinib, lapatinib, neratinib, pazopanib, and sunitinib [35]. We found a similar IC $\mathrm{I}_{50}$ for gefinitib inhibition of MRP4 (4.48 $\left.\mu \mathrm{M}\right)$ to that of Morgan, et al. $(4.6 \mu \mathrm{M})$, and a somewhat comparable $\mathrm{IC}_{50}$ for dasatanib $(18.03 \mu \mathrm{M}$ in the present study versus $27.3 \mu \mathrm{M}$ ). However, the $\mathrm{IC}_{50}$ values for the remaining TKIs evaluated by Morgan, et al. were notably different, which may reflect differences in the experimental conditions such as the specific transporter substrate and range of inhibitor concentrations selected for investigation (e.g. extrapolation of an $\mathrm{IC}_{50}$ value beyond the tested concentrations) $[35,36]$. The majority of the potent MRP4 inhibitors fell within the EGFR TKI class with only one potent inhibitor each in the dual VEGFR \& PDGFR and the BCR/ABL classes. Four AKIs were tested for their potential to inhibit MRP4 (Figure 3). Two AKIs, danusertib and tozasertib, produced IC $\mathrm{C}_{50}$ values $<10 \mu \mathrm{M}$ (Table 2). In particular, tozasertib exhibited comparable inhibitory potency to MK571 (1.87 $\pm 0.81 \mu \mathrm{M}$ versus $2.04 \mu \mathrm{M}$, respectively). In total, eight of the $20 \mathrm{TKI} / \mathrm{AKI}$ assessed were identified as potent inhibitors $\left(\mathrm{IC}_{50} \leq 10 \mu \mathrm{M}\right)$ of MRP4.

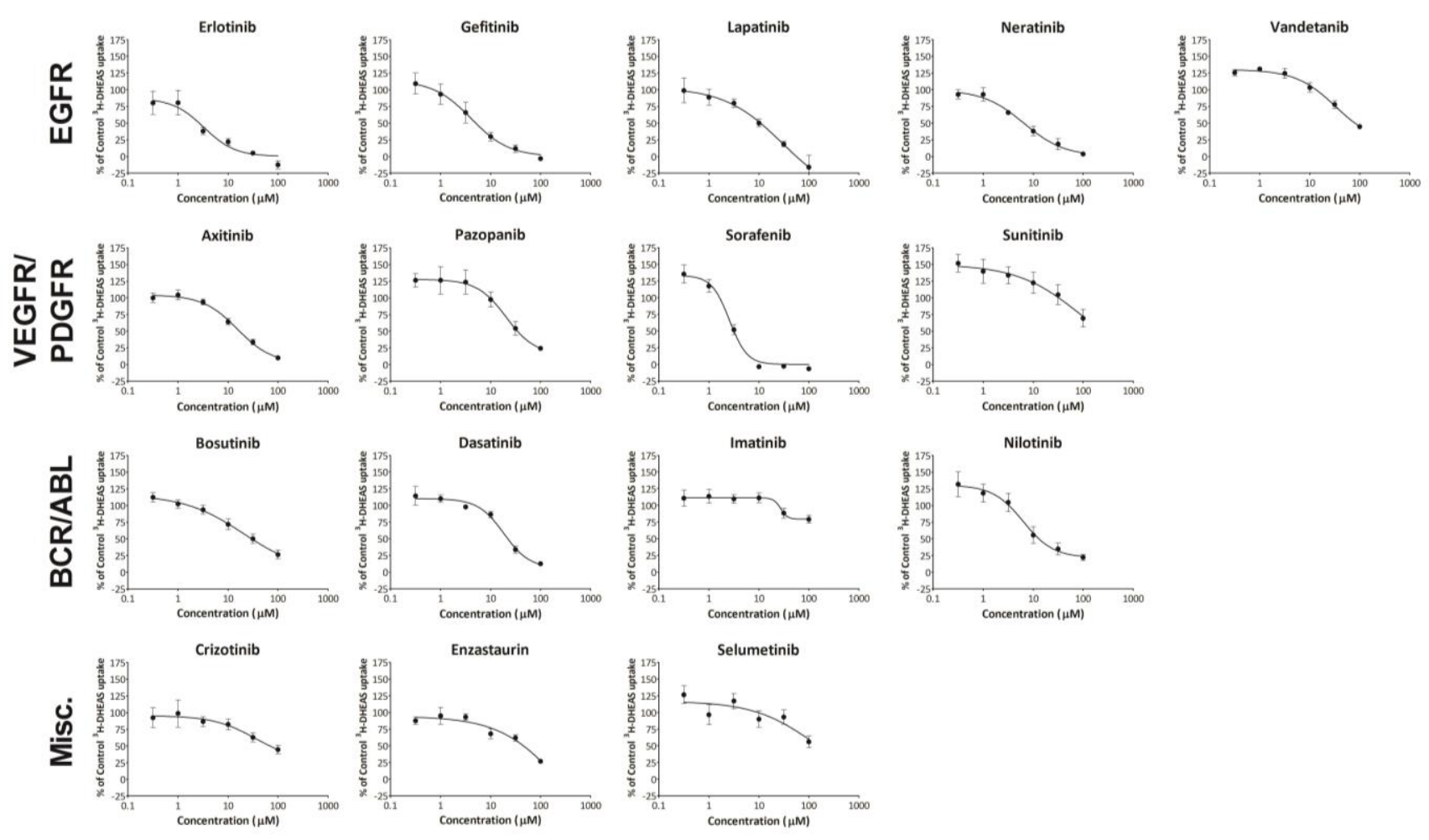

Figure 2. MRP4 Inhibition by Tyrosine Kinase Inhibitors. Inhibition of MRP4-mediated [ $\left.{ }^{3} \mathrm{H}\right]-\mathrm{DHEAS}(2 \mu \mathrm{M} ; 3.5$ min) uptake across an inhibitor concentration range of $0.316-100 \mu \mathrm{M}$ by EGFR, VEGFR/PDGFR, BCR/ABL inhibitors, and inhibitors with miscellaneous targets is shown. Data are presented as mean $\%$ of control uptake $\pm S D, n=3-6$ independent experiments.

BCRP over-expression in MDCKII-BCRP membrane vesicles was determined via western blot analysis and compared to control MDCKII vesicles (Figure 4A). BCRP over-expression was confirmed to be substantially greater in MDCKII-BCRP vesicles. To establish the BCRP membrane vesicle transport assay, the time- and ATP-dependent uptake of $\left[{ }^{3} \mathrm{H}\right]-\mathrm{E} 1 \mathrm{~S}$ was measured in MDCKII and MDCKII-BCRP vesicles (Figure 4A). The uptake of $\left[{ }^{3} \mathrm{H}\right]$-E1S was linear up to 1.5 minutes and appeared to saturate by 3 minutes. An incubation time of 1.5 minutes was chosen for subsequent assessment of potential BCRP inhibitors. There was at least a 35fold increase in $\left.{ }^{3} \mathrm{H}\right]$-E1S uptake in MDCKII-BCRP versus MDCKII vesicles. Several compounds ascribed as BCRP inhibitors were assessed for their potential as positive controls in the membrane vesicle inhibition assay. Sulfasalazine and promethazine were discovered to have the most consistent inhibition profile across multiple independent experiments $(n=9-12)$. The dose-response curves for sulfasalazine and 
promethazine are shown in Figure 4B. Promethazine exhibited an $\mathrm{IC}_{50}$ value of $9.83 \mu \mathrm{M}$ (95\% confidence interval: 5.14-18.80 $\mu \mathrm{M}$ ), while sulfasalazine proved to be a much more potent inhibitor of BCRP with an $\mathrm{IC}_{50}$ value of $0.79 \mu \mathrm{M}$ (95\% confidence interval: 0.64-0.99 $\mu \mathrm{M}$ ). Others have reported an $\mathrm{IC}_{50}$ of $0.6 \mu \mathrm{M}$ for sulfasalazine-mediated inhibition of ${ }^{3} \mathrm{H}$-estrone-3-sulfate uptake in BCRP membrane vesicles derived from HEK-BCRP cells [37].
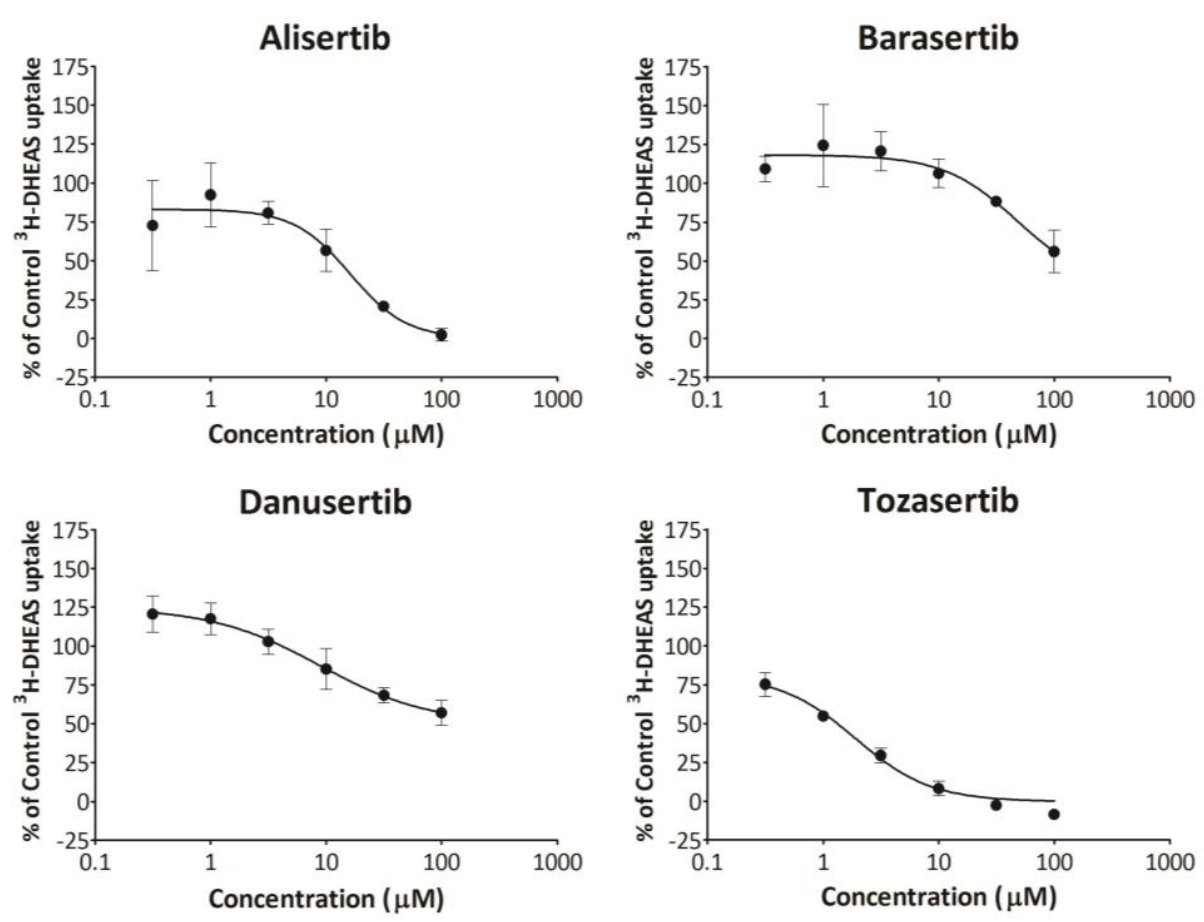

Figure 3. MRP4 Inhibition by Aurora Kinase Inhibitors. Inhibition of MRP4-mediated [ $\left.{ }^{3} \mathrm{H}\right]-\mathrm{DHEAS}(2 \mu \mathrm{M} ; 3.5$ $\mathrm{min}$ ) uptake across an inhibitor concentration range of $0.316-100 \mu \mathrm{M}$ is shown. Data are presented as mean $\%$ of control uptake $\pm S D, n=3-6$ independent experiments.

(A)

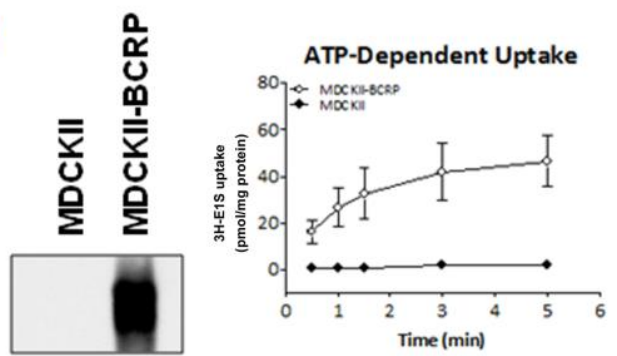

(B)

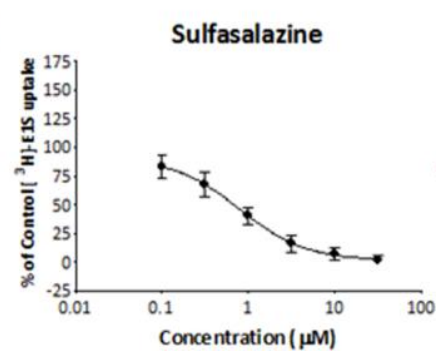

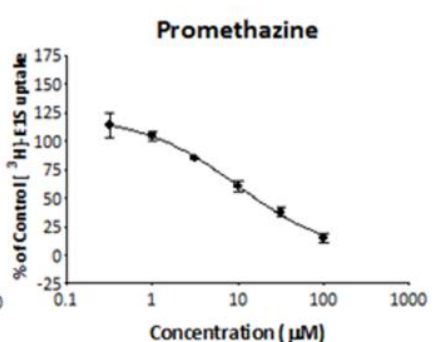

Figure 4. Characterization of the BCRP Membrane Vesicle Assay. (A) BCRP expression in MDCKII and MDCKII$B C R P$ cells. Also shown is ATP-dependent, time-dependent uptake of $1 \mu \mathrm{M}\left[{ }^{3} \mathrm{H}\right]$-estrone sulfate $(\mathrm{E} 1 \mathrm{~S})$ in membrane vesicles. Data are presented as mean $\pm S D, n=3$ independent experiments. (B) Dose-response $I_{50}$ curves of the BCRP positive control inhibitors sulfasalazine and promethazine are shown (1.5 min incubation). Data are presented as mean $\%$ of control uptake $\pm S D, n=9-12$ independent experiments.

Of the 16 TKIs assessed, the majority (the EGFR inhibitors erlotinib, gefitinib, neratinib and vandetanib; the VEGFR \& PDGFR inhibitors axitinib, pazopanib, sorafenib and sunitinib; the BCR/ABL inhibitors bosutinib, imatinib and nilotinib; and the protein kinase $C$ isoform inhibitor enzastaurin) were found to be potent inhibitors of BCRP with $\mathrm{IC}_{50}$ values $<10 \mu \mathrm{M}$ (Figure 5). Notably, erlotinib, gefitinib, sorafenib, imatinib, nilotinib, and enzaustaurin produced $\mathrm{IC}_{50}$ values $<1 \mu \mathrm{M}$. A previous report identified axitinib as 
only a partial inhibitor of BCRP with an $\mathrm{IC}_{50}$ value of $4.4 \mu \mathrm{M}$; however the experiments were performed in MCDK-BCRP cell monolayers and may not be directly comparable to isolated membrane vesicle systems [14]. TKI inhibition potency towards BCRP was not overrepresented in one inhibitor class versus another as was seen for EGFR inhibitors with respect to MRP4. One TKI, lapatinib, did not produce a dose-response curve and was deemed to not be an inhibitor of BCRP. Dose-response curves for AKI inhibition of BCRPmediated transport are shown in Figure 6. In the AKI class, alisertib, barasertib, and danusertib were identified as potent inhibitors of BCRP with $I_{50}$ values $<10 \mu \mathrm{M}$ (Table 2). Unlike the TKIs, none of the AKIs produced $\mathrm{IC}_{50}$ values $<1 \mu \mathrm{M}$ and thus appeared to be slightly less potent inhibitors of BCRP.

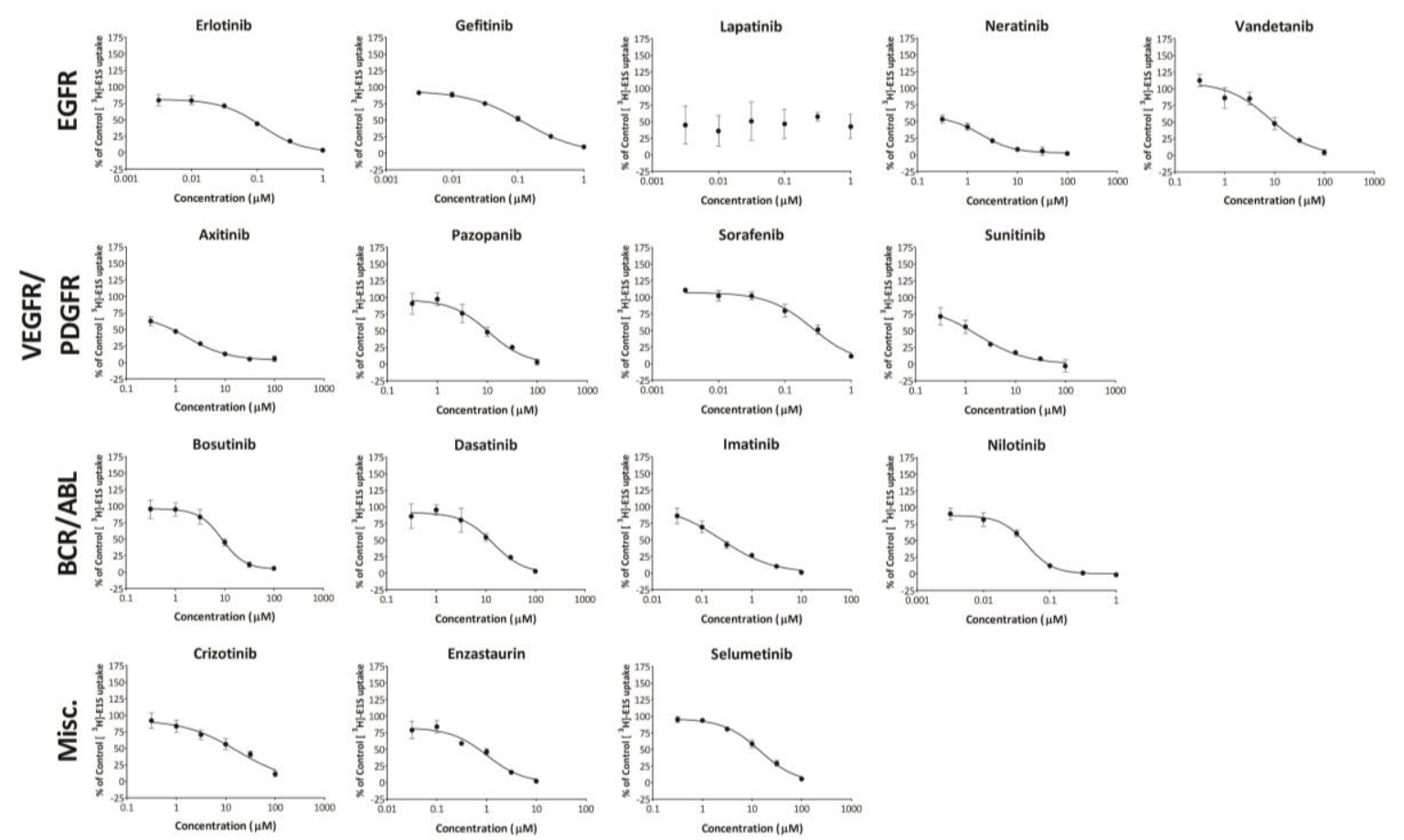

Figure 5. BCRP Inhibition by Tyrosine Kinase Inhibitors. Inhibition of BCRP-mediated $\left[{ }^{3} \mathrm{H}\right]-\mathrm{E} 1 \mathrm{~S}(1 \mu \mathrm{M} ; 1.5 \mathrm{~min})$ uptake across an inhibitor concentration range of $0.00316-100 \mu \mathrm{M}(0.00316-1 \mu \mathrm{M}$ or 0.0316-10 $\mu \mathrm{M}$ for potent inhibitors) by EGFR, VEGFR/PDGFR, BCR/ABL inhibitors, and inhibitors with miscellaneous targets is shown. Data are presented as mean $\%$ of control uptake $\pm S D, n=3-7$ independent experiments. 

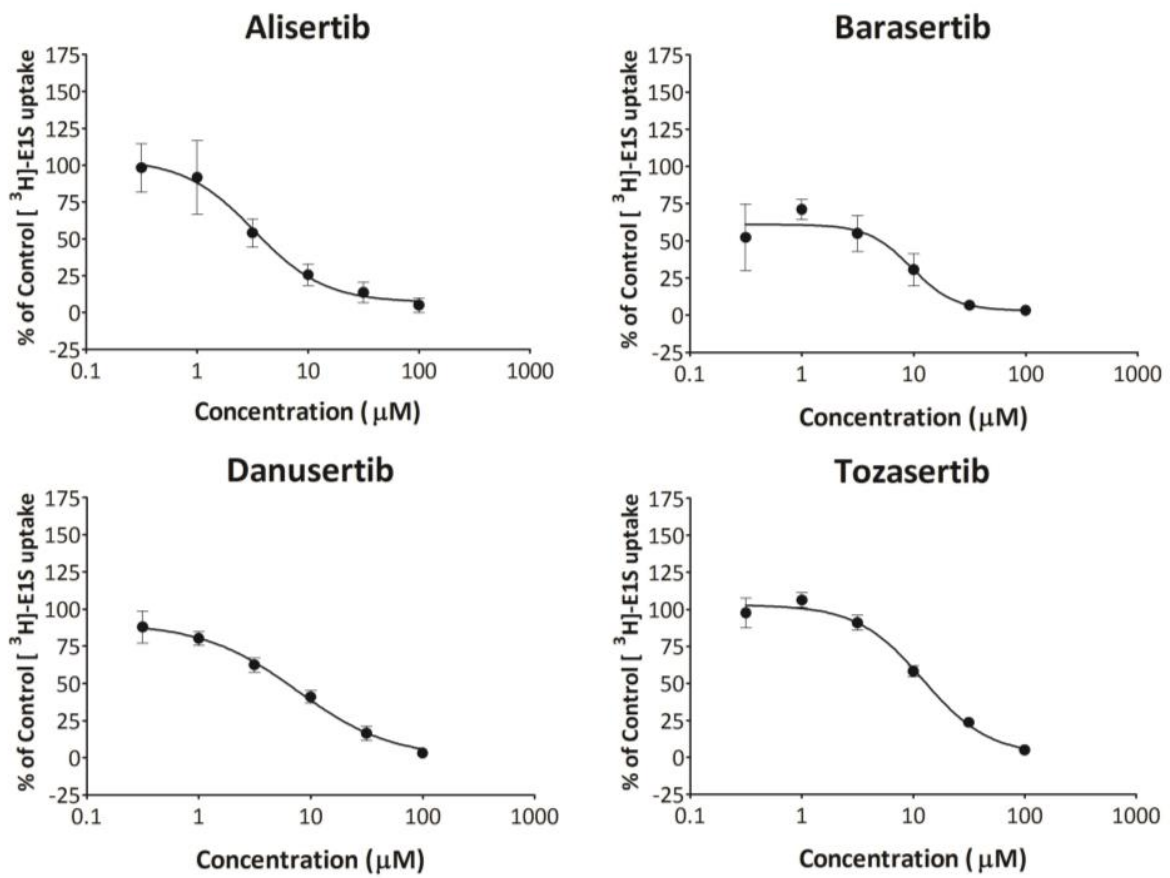

Figure 6. BCRP Inhibition by Aurora Kinase Inhibitors. Inhibition of BCRP-mediated [ $\left.{ }^{3} \mathrm{H}\right]-\mathrm{E} 1 \mathrm{~S}(1 \mu \mathrm{M} ; 1.5 \mathrm{~min})$ uptake across an inhibitor concentration range of 0.316-100 $\mu \mathrm{M}$ is shown. Data are presented as mean $\%$ of control uptake $\pm S D, n=3-4$ independent experiments.

To provide insight to the clinical relevance of TKI/AKI inhibition of MRP4- and BCRP-mediated transport, steady-state total plasma $C_{s s}$ values were extracted from Micromedex and manufacturer package inserts. The $C_{s s}$ values were expressed as a ratio to the $I C_{50}$ for each transporter with a $C_{s s} / I C_{50}$ ratio $>0.1$ regarded as having potential for clinically relevant transporter inhibition [35, 38]. Although it is well-recognized that drug concentrations within cells may become more concentrated than measured $C_{\mathrm{ss}}$ in patient plasma, either due to transporter-mediated accumulation or organelle binding, the use of total plasma $C_{\mathrm{ss}}$ values have been recommended and are used herein in the absence of unbound intracellular drug concentration data $[35,38,39] . C_{s s} / I C_{50}$ ratios are shown in Table 2 . Nine of the $20 \mathrm{TKI} / \mathrm{AKI}$ tested exhibited $C_{\text {ss }} / \mathrm{IC}_{50}$ ratios $>0.1$ for MRP4. In particular, alisertib, danusertib, erlotinib, lapatinib, neratinib, nilotinib, pazopanib, sorafenib, and tozasertib were identified as having potential for clinically relevant MRP4 inhibition. Although the majority of potent MRP4 inhibitors ( $\left.I C_{50} \leq 10 \mu \mathrm{M}\right)$ were found to be in the EGFR inhibitor class, the TKI/AKIs identified as having potential for clinical relevance were primarily stratified across both EGFR and Aurora kinase inhibitor classes. Only 2 VEGFR \& PDGFR and 1 BCR/ABL kinase inhibitors were found to be clinically relevant. Similar to the report of Morgan, et al., pazopanib was identified as having the potential for clinically relevant MRP4 inhibition [35]. Additionally, though the studies of Morgan, et al., also identified gefitinib as a potent inhibitor of MRP4, gefitinib-mediated inhibition of MRP4 transport activity was not deemed clinically relevant [35], which is similar to the current results. The majority of the TKI/AKIs investigated were found to potentially be clinically relevant inhibitors of BCRP, with 15 of the 20 compounds having a $C_{s s} / I C_{50}$ ratio $>0.1$. Specific compounds identified were alisertib, barasertib, danusertib, enzastaurin, erlotinib, gefitinib, imatinib, neratinib, nilotinb, pazopanib, selumetinib, sorafenib, sunitinib, tozasertib, and vandetanib. Several compounds, notably alisertib, danusertib, erlotinib, neratinib, nilotinib, pazopanib, sorafenib, and tozasertib were found to be dual inhibitors of MRP4 and BCRP with potential clinical relevance for both. Dual inhibition may further complicate drug-mediated transporter inhibition by minimizing compensatory mechanisms for export of compounds from within a cell. In summation, the analysis of $C_{\mathrm{ss}} / \mathrm{IC}_{50}$ ratios revealed a greater potential for clinically relevant BCRP inhibition 
by TKI/AKIs compared to MRP4, though eight TKI/AKIs were found to be dual inhibitors of both transporters.

Table 2. Summary of TKI and AKI Inhibition of MRP4 and BCRP. Data are presented as mean relative $\mathrm{IC}_{50}$ estimates $(\mu \mathrm{M})$. Total plasma $C_{\max }$ values $(\mu \mathrm{M})$ were extracted from Micromedex 2.0 (Truven Health Analytics) and manufacturer package inserts. N/A $=$ data not available. Asterisk $(*)$ indicates $C_{\max } / I_{50}$ ratio $>0.1$ for an individual transporter.

\begin{tabular}{|c|c|c|c|c|c|}
\hline TKI/AKI & $\begin{array}{l}\text { Steady-State } \\
\text { Total } C_{s s}(\mu M)\end{array}$ & $\begin{array}{l}\text { Mean Relative MRP4 IC } 50 \\
\text { Estimate }(\mu \mathrm{M}, \pm \mathrm{SD})\end{array}$ & $\begin{array}{l}\mathrm{C}_{\mathrm{ss}} / \mathrm{IC}_{50} \\
\mathrm{MRP4}\end{array}$ & $\begin{array}{c}\text { Mean Relative BCRP IC } 50 \\
\text { Estimate }(\mu \mathrm{M}, \pm \mathrm{SD})\end{array}$ & $\begin{array}{l}\mathrm{C}_{s s} / \mathrm{IC}_{50} \\
\mathrm{BCRP}\end{array}$ \\
\hline Alisertib & 1.80 & $16.54( \pm 4.34)$ & $0.109 *$ & $3.00( \pm 0.38)$ & $0.600^{*}$ \\
\hline Axitinib & 0.07 & $14.99( \pm 5.29)$ & 0.005 & $1.56( \pm 1.07)$ & 0.045 \\
\hline Barasertib & 2.30 & $86.30( \pm 81.93)$ & 0.027 & $7.62( \pm 7.61)$ & $0.302 *$ \\
\hline Bosutinib & 0.38 & $16.75( \pm 14.88)$ & 0.023 & $9.11( \pm 1.81)$ & 0.042 \\
\hline Crizotinib & 1.09 & $\begin{array}{c}\text { N/A (outside } \\
\text { concentration range) }\end{array}$ & N/A & $15.90( \pm 8.23)$ & 0.069 \\
\hline Danusertib & 9.34 & $8.70( \pm 5.18)$ & $1.074^{*}$ & $7.49( \pm 2.70)$ & $1.248^{*}$ \\
\hline Dasatinib & 0.13 & $18.03( \pm 4.42)$ & 0.007 & $13.17( \pm 2.50)$ & 0.010 \\
\hline Enzastaurin & 2.19 & $43.93( \pm 23.77)$ & 0.050 & $0.97( \pm 0.34)$ & $2.266^{*}$ \\
\hline Erlotinib & 3.95 & $3.11( \pm 1.16)$ & $1.270^{*}$ & $0.11( \pm 0.02)$ & $36.127^{*}$ \\
\hline Gefitinib & 0.43 & $4.48( \pm 1.64)$ & 0.096 & $0.12( \pm 0.01)$ & $3.728^{*}$ \\
\hline Imatinib & 2.72 & N/A (partial inhibitor) & N/A & $0.29( \pm 0.15)$ & $9.292 *$ \\
\hline Lapatinib & 4.18 & $8.33( \pm 5.68)$ & $0.502 *$ & N/A & N/A \\
\hline Neratinib & 2.95 & $6.65( \pm 0.66)$ & $0.444 *$ & $1.77( \pm 1.44)$ & $1.669 *$ \\
\hline Nilotinib & 3.85 & $6.18( \pm 4.82)$ & $0.623^{*}$ & $0.05( \pm 0.01)$ & 81.828* \\
\hline Pazopanib & 122.58 & $22.92( \pm 13.82)$ & $5.349 *$ & $9.43( \pm 2.85)$ & $13.000^{*}$ \\
\hline Selumetinib & 2.08 & $103.61( \pm 18.42)$ & 0.020 & $15.04( \pm 6.21)$ & $0.138^{*}$ \\
\hline Sorafenib & 21.51 & $2.60( \pm 0.33)$ & $8.261^{*}$ & $0.26( \pm 0.08)$ & $82.078^{*}$ \\
\hline Sunitinib & 0.23 & $91.53( \pm 40.46)$ & 0.003 & $1.93( \pm 0.37)$ & $0.119 *$ \\
\hline Tozasertib & 2.67 & $1.87( \pm 0.81)$ & $1.427 *$ & $13.74( \pm 3.98)$ & $0.194 *$ \\
\hline Vandetanib & 1.93 & $33.18( \pm 18.90)$ & 0.058 & $8.70( \pm 3.20)$ & $0.222^{*}$ \\
\hline
\end{tabular}

\section{Conclusions}

Together, these data represent the first analysis of the inhibitory potential of multiple TKI/AKIs against both MRP4 and BCRP, along with estimation of the clinical relevance of TKI/AKI-facilitated transport inhibition. The data herein may enable greater predictive power of the pharmacokinetic/pharmacodynamic and potential hepatotoxic effects of TKI/AKI use in co-treatment regimens.

\section{Acknowledgements}

This work was supported by the National Institute of General Medical Sciences of the National Institutes of Health (NIH) under Award Number R01 GM041935. Dr. Hardwick was supported by the National Institute of Environmental Health Sciences under Award Number T32 ES007126. Dr. Ferslew was supported by an Amgen Predoctoral Fellowship in Pharmacokinetics and Drug Disposition. MDCKII and MDCKII-BCRP cell lines were a generous gift from Dr. Markus Grube, Ernst Moritz Arndt University of Greifswald, Greifswald, Germany. HEK-MOCK and HEK-MRP4 cell lines were created by Dr. Kathleen Köck, UNC-CH, using plasmids generously donated by Dr. Dietrich Keppler, German Cancer Research Center, Heidelberg, Germany.

\section{References}

[1] N.P. van Erp, H. Gelderblom, H.J. Guchelaar, Cancer treatment reviews 35 (2009) 692-706.

[2] J. Zhang, P.L. Yang, N.S. Gray, Nature reviews. Cancer 9 (2009) 28-39.

[3] A. Arora, E.M. Scholar, The Journal of pharmacology and experimental therapeutics 315 (2005) 971979. 
[4] S. Niijima, A. Shiraishi, Y. Okuno, Journal of chemical information and modeling 52 (2012) 901-912.

[5] J. Andrae, R. Gallini, C. Betsholtz, Genes \& development 22 (2008) 1276-1312.

[6] R.A. Bradshaw, R.J. Chalkley, J. Biarc, A.L. Burlingame, Advances in biological regulation 53 (2013) 8796.

[7] M. Kollareddy, P. Dzubak, D. Zheleva, M. Hajduch, Biomedical papers of the Medical Faculty of the University Palacky, Olomouc, Czechoslovakia 152 (2008) 27-33.

[8] M.R. Green, J.E. Woolery, D. Mahadevan, Expert opinion on drug discovery 6 (2011) 291-307.

[9] J.T. Hartmann, M. Haap, H.G. Kopp, H.P. Lipp, Current drug metabolism 10 (2009) 470-481.

[10] A. Azzariti, L. Porcelli, G.M. Simone, A.E. Quatrale, N.A. Colabufo, F. Berardi, R. Perrone, M. Zucchetti, M. D'Incalci, J.M. Xu, A. Paradiso, Cancer chemotherapy and pharmacology 65 (2010) 335-346.

[11] A. Brozik, C. Hegedus, Z. Erdei, T. Hegedus, C. Ozvegy-Laczka, G. Szakacs, B. Sarkadi, Expert opinion on drug metabolism \& toxicology 7 (2011) 623-642.

[12] C. Ozvegy-Laczka, J. Cserepes, N.B. Elkind, B. Sarkadi, Drug resistance updates : reviews and commentaries in antimicrobial and anticancer chemotherapy 8 (2005) 15-26.

[13] M. Galetti, P.G. Petronini, C. Fumarola, D. Cretella, S. La Monica, M. Bonelli, A. Cavazzoni, F. Saccani, C. Caffarra, R. Andreoli, A. Mutti, M. Tiseo, A. Ardizzoni, R.R. Alfieri, PloS one 10 (2015) e0141795.

[14] E.L. Reyner, S. Sevidal, M.A. West, A. Clouser-Roche, S. Freiwald, K. Fenner, M. Ullah, C.A. Lee, B.J. Smith, Drug metabolism and disposition: the biological fate of chemicals 41 (2013) 1575-1583.

[15] X.K. Wang, L.W. Fu, Current drug metabolism 11 (2010) 618-628.

[16] S. Shukla, R.W. Robey, S.E. Bates, S.V. Ambudkar, Drug metabolism and disposition: the biological fate of chemicals 37 (2009) 359-365.

[17] Y. Wei, Y. Ma, Q. Zhao, Z. Ren, Y. Li, T. Hou, H. Peng, Molecular cancer therapeutics 11 (2012) 16931702.

[18] C. Hegedus, K. Truta-Feles, G. Antalffy, G. Varady, K. Nemet, C. Ozvegy-Laczka, G. Keri, L. Orfi, G. Szakacs, J. Settleman, A. Varadi, B. Sarkadi, Biochemical pharmacology 84 (2012) 260-267.

[19] N.D. Pfeifer, R.N. Hardwick, K.L. Brouwer, Annual review of pharmacology and toxicology 54 (2014) 509-535.

[20] C.D. Klaassen, L.M. Aleksunes, Pharmacological reviews 62 (2010) 1-96.

[21] K. Kock, K.L. Brouwer, Clinical pharmacology and therapeutics 92 (2012) 599-612.

[22] F.J. Sharom, Pharmacogenomics 9 (2008) 105-127.

[23] Y. An, W.M. Ongkeko, Expert opinion on drug metabolism \& toxicology 5 (2009) 1529-1542.

[24] M. Dohse, C. Scharenberg, S. Shukla, R.W. Robey, T. Volkmann, J.F. Deeken, C. Brendel, S.V. Ambudkar, A. Neubauer, S.E. Bates, Drug metabolism and disposition: the biological fate of chemicals 38 (2010) 1371-1380.

[25] A. Argiris, M. Ghebremichael, J. Gilbert, J.W. Lee, K. Sachidanandam, J.M. Kolesar, B. Burtness, A.A. Forastiere, Journal of clinical oncology : official journal of the American Society of Clinical Oncology 31 (2013) 1405-1414.

[26] L.G. Paz-Ares, B. Biesma, D. Heigener, J. von Pawel, T. Eisen, J. Bennouna, L. Zhang, M. Liao, Y. Sun, S. Gans, K. Syrigos, E. Le Marie, M. Gottfried, J. Vansteenkiste, V. Alberola, U.P. Strauss, E. Montegriffo, T.J. Ong, A. Santoro, N.R.E.U.S.I.S. Group, Journal of clinical oncology : official journal of the American Society of Clinical Oncology 30 (2012) 3084-3092.

[27] T.K. Choueiri, R.W. Ross, S. Jacobus, U. Vaishampayan, E.Y. Yu, D.I. Quinn, N.M. Hahn, T.E. Hutson, G. Sonpavde, S.C. Morrissey, G.C. Buckle, W.Y. Kim, D.P. Petrylak, C.W. Ryan, M.A. Eisenberger, A. Mortazavi, G.J. Bubley, M.E. Taplin, J.E. Rosenberg, P.W. Kantoff, Journal of clinical oncology : official journal of the American Society of Clinical Oncology 30 (2012) 507-512.

[28] K.T. Flaherty, S.J. Lee, F. Zhao, L.M. Schuchter, L. Flaherty, R. Kefford, M.B. Atkins, P. Leming, J.M. Kirkwood, Journal of clinical oncology : official journal of the American Society of Clinical Oncology 31 (2013) 373-379. 
[29] K. Kock, B.C. Ferslew, I. Netterberg, K. Yang, T.J. Urban, P.W. Swaan, P.W. Stewart, K.L. Brouwer, Drug metabolism and disposition: the biological fate of chemicals 42 (2014) 665-674.

[30] M.A. Welch, K. Kock, T.J. Urban, K.L. Brouwer, P.W. Swaan, Drug metabolism and disposition: the biological fate of chemicals 43 (2015) 725-734.

[31] M. Grube, S. Reuther, H. Meyer Zu Schwabedissen, K. Kock, K. Draber, C.A. Ritter, C. Fusch, G. Jedlitschky, H.K. Kroemer, Drug metabolism and disposition: the biological fate of chemicals 35 (2007) 30-35.

[32] K. Kock, A. Koenen, B. Giese, M. Fraunholz, K. May, W. Siegmund, E. Hammer, U. Volker, G. Jedlitschky, H.K. Kroemer, M. Grube, The Journal of biological chemistry 285 (2010) 11336-11347.

[33] G. Ghibellini, E.M. Leslie, G.M. Pollack, K.L. Brouwer, Pharmaceutical research 25 (2008) 1851-1860.

[34] B.C. Ferslew, K. Kock, A.S. Bridges, K.L. Brouwer, Drug metabolism and disposition: the biological fate of chemicals 42 (2014) 1567-1574.

[35] R.E. Morgan, C.J. van Staden, Y. Chen, N. Kalyanaraman, J. Kalanzi, R.T. Dunn, 2nd, C.A. Afshari, H.K. Hamadeh, Toxicological sciences : an official journal of the Society of Toxicology (2013).

[36] C.J. van Staden, R.E. Morgan, B. Ramachandran, Y. Chen, P.H. Lee, H.K. Hamadeh, Current protocols in toxicology / editorial board, Mahin D. Maines Chapter 23 (2012) Unit 2325.

[37] J.E. Karlsson, C. Heddle, A. Rozkov, J. Rotticci-Mulder, O. Tuvesson, C. Hilgendorf, T.B. Andersson, Drug metabolism and disposition: the biological fate of chemicals 38 (2010) 705-714.

[38] M.J. Zamek-Gliszczynski, C.A. Lee, A. Poirier, J. Bentz, X. Chu, H. Ellens, T. Ishikawa, M. Jamei, J.C. Kalvass, S. Nagar, K.S. Pang, K. Korzekwa, P.W. Swaan, M.E. Taub, P. Zhao, A. Galetin, C. International Transporter, Clinical pharmacology and therapeutics 94 (2013) 64-79.

[39] N.D. Pfeifer, K.B. Harris, G.Z. Yan, K.L. Brouwer, Drug metabolism and disposition: the biological fate of chemicals (2013).

(C2016 by the authors; licensee IAPC, Zagreb, Croatia. This article is an open-access article distributed under the terms and conditions of the Creative Commons Attribution license (http://creativecommons.org/licenses/by/3.0/) (cc)) Er 